\title{
Preface: celebrating the 60th anniversary of the University of Science and Technology of China
}

\author{
Jinlong Yang ${ }^{*} \&$ Tongwen $\mathrm{Xu}^{*}$ \\ School of Chemistry and Materials Science, University of Science and Technology of China, Hefei 230026, China
}

Received July 18, 2018; accepted July 18, 2018; published online August 3, 2018

Citation: Yang JL, Xu TW. Preface: celebrating the 60th anniversary of the University of Science and Technology of China. Sci China Chem, 2018, 61: 1043-1044, https://doi.org/10.1007/s11426-018-9338-5

The University of Science and Technology of China (USTC) is a prominent institution with an excellent reputation for education and innovation that resonates across the world. The university holds a special place in history. It was established by the Chinese Academy of Sciences (CAS) in 1958 to meet China's science and technology needs and increase the country's international competitiveness. Throughout its history we have focused on frontier areas of science and technology and educated top leaders in science and technology for China and the world.

USTC has always been at the front line of chemistry research and education in China. The founders of these chemical related subjects include Dagang Liu, Yonghuai Guo, Xuesen Qian, Chengzong Yang, Baoren Wang, Renyuan Qian, and so on, who are outstanding and wellknown scientists in China. The School of Chemistry and Materials Science was created in 1996 by the amalgamation of Department of Chemical Physics, Department of Applied Chemistry, and Department of Materials Sciences and Engineering. In its recent form, the school now consists of 5 departments (Department of Chemistry, Department of Chemical Physics, Department of Applied Chemistry, Department of Materials Sciences and Engineering, and Department of Polymer Science and Engineering) and one center (Chemical Experimental Teaching Center), and covers 2 first-tier disciplines, chemistry and materials science; and 12 sub-disciplines, including inorganic chemistry, ana-

*Corresponding authors (email: jlyang@ustc.edu.cn; twxu@ustc.edu.cn) lytical chemistry, organic chemistry, physical chemistry (including chemical physics), polymer chemistry and physics, renewable and clean energy, applied chemistry, chemical biology, energy chemistry, materials physics and chemistry, materials science, and materials processing engineering. The school embraces 3 key laboratories of CAS, and 2 key laboratories of Anhui province. It also takes part in 3 collaborative innovation centers of Ministry of Education (MOE). Currently, the school has 104 professors and 43 associate-professors, including 17 academicians of CAS/CAE (with adjunct faculties).

At the School of Chemistry and Materials Science, we conduct a wide range of high quality researches. The school emphasizes frontier and interdisciplinary sciences, and highlights the joint development of fundamental and applied researches. Aiming at tackling some of the biggest challenges faced by both China and the world such as energy \& resources problems, and population \& health problems, our school has taken on lots of national research projects, and publishes more than 600 SCI papers and tens of patents each year. In the 2018 Nature Index top institution ranking in Chemistry, USTC ranked 6th among global institutions. From 2010 to 2017, the school had earned 6 National Natural Science Awards (second prize), 6 Science \& Technology Awards of Anhui Province (first prize), and 2 Natural Science Awards of the Ministry of Education (first prize). In the fourth Chinese University Ranking by Disciplines carried out in 2017 by MOE, Chemistry and Material Science and Engineering of USTC got A+ and A- rankings, respectively. 
The school is well recognized throughout the world for the quality of teaching. It is nominated as "National Base for Talents in Basic Scientific Research and Education", and its Chemical Experimental Teaching Center is chosen as the national demonstration center. It has been awarded 12 "National Excellent 100 Doctoral Dissertation" prizes. More than $80 \%$ of the undergraduates pursue further education in universities and institutes either at home or abroad. Over the years, the school has fostered large numbers of outstanding talents in different fields for the sustainable development of China as well as the world.

In the celebration of the 60th anniversary of USTC, we organized special issues, which include 13 articles in Chi- nese special issue and 15 articles in English special issue, relating to recent accomplishments in the fields of theoretical and computational chemistry, solid-state chemistry, organic chemistry, analytical chemistry and applied chemistry from our school. We expect exchange of ideas and experiences with researchers in China and abroad, foster new research collaborations, and promote sustaining development in chemistry. We would like to thank Prof. Li-Jun Wan and his colleagues from the editorial board of Science China Chemistry very much for their efforts on this special issue. The School of Chemistry and Materials Science of USTC will do her best in scientific research and education for the development of China and the world.

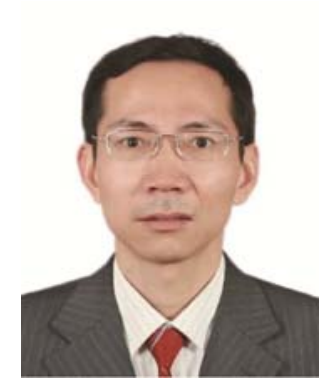

Prof. Jinlong Yang is a full professor of chemistry at the University of Science and Technology of China (USTC). He obtained his Ph.D. degree in 1991 from USTC. He was awarded Outstanding Youth Foundation of China in 2000, selected as Changjiang Scholars Program Chair Professor in 2001 and as a fellow of American Physical Society (APS) in 2011. He is the second awardee of the 2005's National Award for Natural Science (the second prize) and the awardee (principal contributor) of the 2014's Outstanding Science and Technology Achievement Prize of the Chinese Academy of Sciences (CAS). His research mainly focuses on the development of first-principles methods and their application to clusters, nanostructures, solid materials, surfaces, and interfaces.

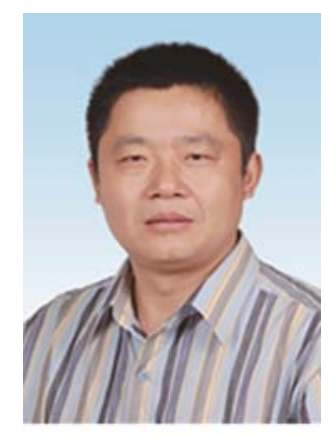

Prof. Tongwen $\mathrm{Xu}$ is a full professor of chemistry at the University of Science and Technology of China. He received his Bachelor's degree in 1989 and Master's degree in 1992 from Hefei University of Technology, China. He received his Ph.D. degree of Chemical Engineering in 1995 from Tianjin University. He was a short-term visiting scientist of University of Tokyo and Tokyo Institute of Technology in 2000 and 2001, respectively. He received a Brain Pool Program from Korea (2006) and worked one year in Gwangju Institute of Science and Technology. He was awarded Outstanding Youth Foundation of China in 2010, selected as Fellow of Royal Society of Chemistry in 2013 and as Changjiang Scholars Program Chair Professor in 2014. Currently, he is working on materials chemical engineering especially on ion exchange membrane materials and the related applications. He has held 60 patents and published 400 SCI papers with over 10000 citations and H-index 54. 